\title{
Osteoporosis: A Disease of Men
}

\author{
Anu Garg1, Samantha Kay² \\ ${ }^{1}$ Department of Medicine, University of Toledo, Toledo, OH, USA \\ ${ }^{2}$ University of Toledo, Toledo, OH, USA \\ Email: anu.garg@utoledo.edu
}

How to cite this paper: Garg, A. and Kay, S. (2018) Osteoporosis: A Disease of Men. Health, 10, 69-74.

https://doi.org/10.4236/health.2018.101005

Received: November 28, 2017

Accepted: January 8, 2018

Published: January 11, 2018

Copyright (๑) 2018 by authors and Scientific Research Publishing Inc. This work is licensed under the Creative Commons Attribution International License (CC BY 4.0).

http://creativecommons.org/licenses/by/4.0/

\begin{abstract}
Objective: The goal of this study was to evaluate osteoporosis screening practices in male patients aged 70 and older. Methods: A survey-based study was carried out over six months at an academic primary care institution in the Midwest. Results: Seventy-nine male patients and fifteen primary care physicians were surveyed. Less than $10 \%$ of males recalled being screened for osteoporosis. Two-thirds of physicians reported regularly screening males for osteoporosis. Conclusion: Elderly male patients are overwhelmingly under-screened and undertreated for osteoporosis.
\end{abstract}

\section{Keywords}

Osteoporosis, Geriatrics, Men's Health

\section{Introduction}

Osteoporosis is a disease of women and men. It is a major cause of morbidity and mortality in both men and women but it is often unrecognized and undertreated in male patients. The mortality rate in men within 1 year of hip fracture is $37.5 \%$, which is $51 \%$ higher than in women [1]. Men steadily lose bone mineral density with aging, and one in five men over 50 will suffer an osteoporotic fracture [2]. Although both the 1-year mortality and the need for institutional care after a hip fracture are higher in men than women, men are less likely to receive investigation or treatment for their underlying osteoporosis.

Both the National Osteoporosis Foundation (NOF) and the Endocrine Society recommend dual-energy X-ray absorptiometry (DEXA) screening for osteoporosis in all men aged 70 or older, as well as for men between the ages of 50 and 69 who have additional risk factors. These risk factors include excessive alcohol use, treatment with glucocorticoids, and hypogonadism, especially that experienced by men receiving androgen deprivation therapy (ADT) for prostate can- 
cer [1]. Clear guidelines exist for osteoporosis screening in women. The available guidelines recommend screening high-risk men; however, screening does not appear to be a standard practice. The objective of our study was to examine osteoporosis screening practices in male patients 70 and older in a university-based primary care setting.

\section{Methods}

A single center, survey-based study was carried out at our academic primary care institution over a period of six months. We surveyed all willing and consenting male patients aged 70 and older. A seven-item questionnaire was distributed in person to male patients at their office appointments (see Appendix for questionnaire). Questionnaire items included history of falls and fractures, testing for osteoporosis, knowledge about risk of bone fractures, desire to learn more about fracture risk, interest in being screened for osteoporosis, willingness to take treatment for osteoporosis, and intake of calcium and vitamin D. In addition, we also surveyed primary care physicians who consented to participate. Primary care physicians were asked to complete a five-item questionnaire (see Appendix for questionnaire). This included whether osteoporosis is a disease of men, women, or both; osteoporosis screening practices; National Osteoporosis Foundation screening recommendations in men; morbidity and mortality gender differences from fractures, use of calcium and vitamin D supplements in physician practices.

Data analysis was done using SPSS. Chi-squared tests were run to compare variables from each set of surveys. Person's correlation coefficient was used to evaluate the significance of these variables. A p-value of $<0.05$ was considered to be statistically significant.

\section{Results}

Seventy-nine patients aged 70 years and older were included in the study after questionnaire completion. Our data revealed that nearly half of the patients had fractured a bone or fallen. However, less than $9 \%$ of them recalled being screened for osteoporosis. Nearly $16 \%$ of those with a history of falls or fractures were tested for osteoporosis compared to only $2 \%$ of those who had no known falls or fractures $(\mathrm{p}=0.04)$. Of the males surveyed, $42 \%$ reported being aware of their risk for osteoporosis and bone fracture. There was a marginally significant difference in males with a history of falls in regards to those who knew about their risk of osteoporosis and those who did not $(\mathrm{p}=0.06)$. One-third of male patients reported that they would like to learn more about osteoporosis and their risks for bone fractures. Nearly half (49\%) would be interested in an osteoporosis screening test if offered by their physicians to decrease fracture risk. Of patients interested in screening, $61 \%$ had a history of falls and fractures. Ninety-seven percent of patients interested in screening would take preventative medication compared to only $13 \%$ of those not interested in screening $(p<0.001)$. 
Compared to those with no fall/fracture history, a significantly higher number of male patients who had ever fallen or fractured a bone would be willing to take osteoporosis treatment $(\mathrm{p}=0.05)$. Over half of patients $(57 \%)$ were taking vitamin D and/or calcium regularly. Patients with a history of falls and/or fracture were actually less likely to be taking vitamin supplementation although this difference was not statistically significant $(\mathrm{p}=0.2)$.

Fifteen primary care physicians completed the questionnaire. The data demonstrated that about half of physicians think osteoporosis is a disease of women while the other half see osteoporosis as a disease affecting both men and women. Women are thought to have a higher morbidity and mortality from osteoporosis by two-thirds of surveyed PCPs although the opposite is true. Eleven of the physicians (73\%) are familiar with the National Osteoporosis Foundation's recommendations for osteoporosis screening in men. All the physicians who do not screen male patients are aware of these guidelines. Only two-thirds of those surveyed screen male patients for osteoporosis but a large majority (87\%) do discuss calcium and vitamin D supplements for bone health with male patients. Every physician who screens male patients for osteoporosis discusses the use of calcium and vitamin D with patients compared to $60 \%$ of physicians who do not regularly screen males $(\mathrm{p}=0.03)$.

\section{Discussion}

Although our physicians are well aware of the screening guidelines, they do not consistently offer screening or treatment options to men as often as they do to women. There is a definite lack of osteoporosis screening in males. Our results revealed that a vast majority of male patients have not been screened for osteoporosis. A recent study evaluating the 10-year osteoporosis screening rate in male patients found merely $11.3 \%$ of men over age 70 had undergone DXA scanning [3]. Low screening rates have several consequences not only prior to osteoporosis complications like fractures but also after they occur too.

Despite a higher prevalence of osteoporosis in women compared to men, men have a higher rate of osteoporosis associated complications, including increased morbidity and mortality after hip fracture. This is in large part due to both poor medical management upon discharge and low rates of osteoporosis screening post-fracture [4]. A large single hospital study found that only $5.4 \%$ of men were ordered bone mineral density measurement post-discharge [5]. Treatment with bisphosphonates was shown to independently decrease mortality after hip fracture in older patients with a $60 \%$ reduction in mortality per year of use [6]. However, men are not receiving these treatments. Another study found that at hospital discharge, only $6.8 \%$ of men were receiving any type of osteoporosis treatment after atraumatic hip fracture compared to $31 \%$ of women. One to five years after discharge for hip fracture, merely $9 \%$ of men were receiving osteoporosis medical management while $48 \%$ of their female counterparts received treatment [7]. Lack of osteoporosis treatment may be due to discrepancies in 
who should prescribe such medications. A multi-state study of orthopedic surgeons concluded that $50 \%$ of them avoided prescribing osteoporosis medications to patients after hip fracture due to lack of long-term follow-up and concern for medication adverse effects. These orthopedists believed primary care physicians should initiate medical management instead [8]. Regardless of the cause, men are under-treated and under-screened for osteoporosis and have poorer outcomes compared to women.

These results indicate the need for improved osteoporosis screening pre-fracture and post-fracture, which has its own set of unique challenges. The challenging aspects of screening more male patients are an increased workload for clinicians to counsel patients regarding the need for screening, to interpret the results, and to diagnose, to treat, and to monitor the patients. Medicare coverage is also limited for osteoporosis screening in male patients [3]. There will be increased burden on the Radiology Department as well to scan these men, but patients should at least be given an option to choose or refuse the screening.

There are a few limitations to this study. First, no patient characteristics were collected as the purpose of this study was quality improvement. Without this information, no associations could be made between screening rates and age, race/ethnicity, co-morbidities, medications, social history, etc. Secondly, the study was conducted at a single center. Therefore, the sample size was small, which limited the study's power, and the results are not generalizable.

\section{Conclusion}

Physicians are not screening male patients as often as female patients in our primary care clinic. We would like to improve osteoporosis screening in male patients performed by primary care physicians. One consideration is to provide reading material at each appointment to educate male patients over 70 , about improving bone health. Studies have suggested that there is a lack of knowledge among medical professionals about the risks of osteoporosis and subsequent fractures in male patients [7] [9]. Providing educational material about osteoporosis and hip fractures to both patients and their families may make them more active participants in seeking treatment. Another would be adding osteoporosis screening as a quality indicator in the EMR. Risk stratification questions could be integrated into the history and physical examination during the office interview to screen high-risk men as per National Osteoporosis Foundation guidelines. The FRAX calculator could be utilized as well to estimate fracture risk; however, this modality does tend to underestimate fracture risk in men [4]. Overall, it is hoped that a greater awareness of the problem of osteoporosis in men will lead to better treatment, and ultimately to a decrease in fractures and their associated morbidity and mortality.

\section{Acknowledgements}

The authors thank Dr. Sadik Khuder for his assistance with statistical analysis. 


\section{References}

[1] Borg, S., Chopin, F., Hoppe, E., Morel, G., Biver, E. and Laroche, M. (2011) Why and How Should We Investigate Men for Osteoporosis? Joint Bone Spine, 78, S197-S201. https://doi.org/10.1016/S1297-319X(11)70004-5

[2] Sutton, R., Dian, L. and Guy, P. (2011) Osteoporosis in Men: An Underrecognized and Undertreated Problem. BCMJ, 53, 535-540.

[3] Lim, S.Y., Lim, J.H., Nguyen, D., Okamura, R., Amiri, H.M., Calmes, M. and Nugent, K. (2013) Screening for Osteoporosis in Men Aged 70 Years and Older in a Primary Care Setting in the United States. American Journal of Men's Health, 7, 350-354. https://doi.org/10.1177/1557988313478826

[4] Alswat, K.A. (2017) Gender Disparities in Osteoporosis. Journal of Clinical Medicine Research, 9, 382-387. https://doi.org/10.14740/jocmr2970w

[5] Antonelli, M., Einstadter, D. and Magrey, M. (2014) Screening and Treatment of Osteoporosis after Hip Fracture: Comparison of Sex and Race. Journal of Clinical Densitometry, 17, 479-483. https://doi.org/10.1016/j.jocd.2014.01.009

[6] Beaupre, L.A., Morrish, D.W., Hanley, D.A., Maksymowych, W.P., Bell, N.R., Juby, A.G. and Majumdar, S.R. (2011) Oral Bisphosphonates Are Associated with Reduced Mortality after Hip Fracture. Osteoporosis International, 22, 983-991. https://doi.org/10.1007/s00198-010-1411-2

[7] Kiebzak, G.M., Beinart, G.A., Perser, K., Ambrose, C.G., Siff, S.J. and Heggeness, M.H. (2002) Undertreatment of Osteoporosis in Men with Hip Fracture. Archives of Internal Medicine, 162, 2217-2222. https://doi.org/10.1001/archinte.162.19.2217

[8] Skedros, J.G., Holyoak, J.D. and Pitts, T.C. (2006) Knowledge and Opinions of Orthopedic Surgeons Concerning Medical Evaluation and Treatment of Patients with Osteoporotic Fracture. The Journal of Bone and Joint Surgery, 88, 18-24.

[9] Rao, S.S., Budhwar, N. and Ashfaque, A. (2010) Osteoporosis in Men. American Family Physician, 82, 503-508. 


\section{Appendix}

\section{A1. Patient Questionnaire}

1) Have you ever fallen or fractured a bone?

Yes

No

2) Have you ever been tested for osteoporosis (to check for risk of fracture)?

Yes

No

3) Do you know about your risk of osteoporosis (bone fracture)?

Yes

No

4) Would you be interested in learning more about osteoporosis?

Yes

No

5) Will you be interested in screening test to check for your fracture risk, if offered by your doctor?

Yes

No

6) If the test is positive, would you be willing to take treatment to prevent the fracture?

Yes No

7) Are you taking calcium or vitamin D supplement?

Yes No Either Ca or D

\section{A2. Primary Care Physician Questionnaire}

1) Osteoporosis is primarily a disease of:

Women Men Both

2) Do you screen male patients for osteoporosis?

Yes No

3) Do you know the National Osteoporosis Foundation's age recommendations for osteoporosis screening in men?

Yes No

4) In your opinion, who has higher morbidity and mortality from osteoporosis?

Women

Men

5) Do you discuss the use of calcium and Vitamin D supplements for bone health with male patients?

Yes

No 